\title{
The Myth of When and Where: How False Assumptions Still Haunt Theories of Consciousness
}

\author{
Sepehrdad Rahimian ${ }^{1}$ \\ ${ }^{1}$ Department of Psychology, National Research University Higher School of Economics, \\ Moscow, Russia \\ * Correspondence: \\ Sepehrdad Rahimian \\ srahimian@hse.ru
}

Abstract

Recent advances in neural sciences have uncovered countless facts about the brain. Although there is a plethora of theories of consciousness, it seems to some philosophers that there is still an explanatory gap when it comes to a scientific account of subjective experience. In what follows, I argue why some of our more commonly acknowledged theories do not at all provide us with an account of subjective experience as they are built on false assumptions. These assumptions have led us into a state of cognitive dissonance between maintaining our standard scientific practices on the one hand, and maintaining our folk notions on the other. I end by proposing Illusionism as the only option for a scientific investigation of consciousness and that even if ideas like panpsychism turn out to be holding the seemingly missing piece of the puzzle, the path to them must go through Illusionism.

Keywords: Illusionism, Phenomenal consciousness, Predictive Processing, Consciousness, Cartesian Materialism, Unfolding argument

\section{Introduction}

He once greeted me with the question: "Why do people say that it was natural to think that the sun went round the earth rather than that the earth turned on its axis?"

I replied: "I suppose, because it looked as if the sun went round the earth."

"Well," he asked, "what would it have looked like if it had looked as if the earth turned on its axis?"

-Anscombe, G.E.M (1963), An introduction to Wittgenstein's Tractatus 
In a 2011 paper, Anderson (2011) argued that different theories of attention suffer from arbitrary and often contradictory criteria for defining the term, rendering it more likely that we should be excluding the term "attention" from our scientific theories of the brain. This problem occurs, at least partially, as we try to import our day-to-day concepts into science, while forgetting that "folk psychology" serves a different purpose and should be "interpreted", rather than being taken literally (Dewhurst, 2021). In other words, there may never be a complete and literal one-to-one correspondence between concepts from one domain (e.g. folk psychology) and another (e.g. neuroscience) [Kukla,2017].

The problems faced when defining consciousness are no different, if not worse, given that it has different meaning for different theorists. Here, I use consciousness to refer to the concept of phenomenal consciousness (Block, 1995) or the what-is-it-likeness (Nagel,1974), as it is this aspect of consciousness that is the most controversial. Indeed, there are some who take phenomenal consciousness to be an unfalsifiable notion. For instance, Cohen and Dennett (2011) point out that even a perfect neuroscience experiment, one that perfectly isolates every single neuron involved in causing a subject to report the experience of color, will be unable to capture phenomenal consciousness given that the results of that experiment can be accused of being confounded with various cognitive functions such as decision-making, memory, etc. They write:

What does it mean to study consciousness without function? Inevitably, theories motivated by this view will define consciousness in their own way (local recurrency, microconsciousness, coalitions of neurons, etc.) and say that whenever that criterion is met, consciousness must occur. But how do we set this criterion?

-Cohen and Dennett (2011), p.5

In this contribution, I propose that while the problem of arbitrary criteria does not affect all theories of consciousness equally, even those with the most empirical support suffer from unjustified assumptions from folk psychology.

\section{Structural and Functional Theories of Consciousness}

There are numerous scientific theories of consciousness at hand, but Global Neuronal Workspace Theory (GNWT) [Dehaene \& Naccache, 2001; Mashour, Roelfsema, Changeux, \& Dehaene, 2020], Recurrent Processing Theory (RPT) [Lamme, 2006], Information-Integration Theory (IIT) [Oizumi, Albantakis, \& Tononi, 2014] and recently, the Winning-Hypothesis in predictive coding paradigms (Hohwy,2013) are some of the most famous ones. These theories can be roughly categorized into structural and functional ${ }^{1}$. As the names imply, in structural theories, consciousness is identified with a structure or a particular signal

\footnotetext{
${ }^{1}$ I use structural/functional distinction similar to how Doerig et al. (2019) have used them. This is somewhat different from the more famous and more traditional distinction between Wilhelm Wundt (structuralism) and William James' view (functionalism) on consciousness.
} 
whereas in functional theories, it is treated as an outcome; one which does not need a oneto-one relationship with the structure. (Doerig, Schurger, Hess, \& Herzog, 2019).

For instance, in RPT, consciousness is identified as a recurrent signal (making it a structural theory) whereas in the Winning-Hypothesis account of consciousness, it is identified as a certain top-down hypothesis with the highest posterior probability (Hohwy,2013), defining consciousness in light of a particular function (i.e. guiding behavior through the "best" topdown hypothesis, irrespective of the exact mechanism that has led to generating that hypothesis) and thus, making it a functional theory. Here, I provide two objections that when combined, serve as an opposition to how our current well-established theories go about tackling the mystery of consciousness. Specifically, almost all theories of consciousness have to deal with at least one of these objections.

\subsection{Objection 1: The Unfolding Argument}

Recently, Doerig et al. (2019) raised the unfolding argument against structural theories of consciousness (i.e. IIT and RPT). The argument rests on the premise that both recurrent neural networks and feedforward neural networks are function approximators. Thus, any function that can be carried out by the former, can also be carried out by the latter. This suggests that if consciousness is identified as structural (e.g. a recurrent process [Lamme, 2006]), any input-output function that is carried out by that very process, can also be carried out by a feed-forward network, suggesting that there is nothing inherently special about that particular process or structure that should give rise to conscious processing. Moreover, IIT tracks consciousness by assigning a particular value (i.e. $\Phi$ ) to represent how much integration of information occurs within that system and to see if $\Phi$ has passed a certain threshold. As Doerig et al. point out, for any implementation of an input-output behavior, there are an infinite number of equivalent feedforward and recurrent networks, each capable of producing the exact same results and yet, having a different rate of information integration (i.e. a different $\Phi$ ). This renders it unlikely that any particular $\Phi$ would be necessary for a system to be conscious. (Doerig et al., 2019; but see Tsuchiya, Andrillon, \& Haun, 2019) ${ }^{2}$

\subsection{Objection 2: Cartesian Materialism and Folk Assumptions}

Although the unfolding argument can only be made against structural theories of consciousness, both structural and functional theories can fall victim to having incorrect underlying assumptions. If any functional theory of consciousness identifies certain (physical) function with consciousness, then one can still ask: "what is it about that functional state that makes it feel like something, rather than nothing?". For instance, what is it about the global availability of information (Dehaene \& Naccache, 2001) that makes us feel that we have a non-physical experience?

\footnotetext{
${ }^{2}$ In other words, if the claim is that a particular recurrent signal or a particular $\Phi$ reflects consciousness, you can find other systems that can fulfill the exact same task, while having a different $\Phi$ or being feed-forward rather than recurrent.
} 
It is at this point where we enter philosophical hot water. All theories of consciousness (like any other scientific theory) rest upon various implicit and explicit assumptions that are informed by the empirical findings and shape the direction of future empirical findings. While explicit assumptions are constantly tested by the scientific method, the implicit ones are much harder to evaluate and may be mistakenly treated as facts. Assumptions such as consciousness having a spatial or a temporal onset, being a singular phenomenon, being binary in nature, being the end-product of the brain. Although almost all of these assumptions have been challenged by various philosophers over time, one can still find their fingerprints on our current models of consciousness. My issue here is not with any particular assumption in any given theory so much as it is with the form of thinking that leads to making those assumptions in the first place. The term "Cartesian materialism" has been coined by Dennett (1993) to refer to the instances in which neuroscientists whom if explicitly asked will reject cartesian dualism, use dualistic notions in their text to describe consciousness. While this ironic term elegantly describes how some theories may suffer from unintended dualistic assumptions, I argue that the real problem lies elsewhere; namely, there is a breed of unjustified assumptions (including the dualistic ones) that pose a threat to the progress of the science of consciousness.

As an analogy, imagine there are investigators searching a house to find a man hiding there, based on some eyewitness testimonies. According to one neighbor, the man can be found, hiding behind the door. To verify that, the investigators will take that claim seriously and look behind the door, only to find that there is nobody there. Now imagine the same neighbor, with the same degree of confidence as the last time, coming up with other propositions: "the man is hiding under the bed", "the man is hiding in the kitchen", etc. While all of these claims are testable and while it is possible to build theories around them that look for signs that someone had been at those locations, at some point the investigators may wonder why in the first place do some witnesses believe there is a man hiding somewhere? The problem here is more about the form of thinking (i.e. there is a man hiding somewhere) than it is about any particular hypothesis (e.g. there is a man specifically under the bed or in the kitchen). If there is any chance that this form of thinking is incorrect, at some point we could benefit from changing our thinking template; perhaps there is a woman instead of a man, and she is not hiding, but rather silently reading. Focusing too much on the former way of thinking, keeps us from exploring the possibility of the latter. At some point we must allow our failure to locate the missing man in various locations, lower our confidence in our assumptions (e.g. someone is hiding, that person is a man) and not merely our hypotheses (i.e. the missing man being hidden in a particular place). Moreover, a systematic search to locate the missing man could seem like empirical progress beyond merely exploring the house. And in some cases, different theorists could insist that a particular male statue in the house is indeed our missing man, because exactly as their models predict, you would find a man hiding behind the bedroom door, and what we have found behind the bedroom door looks similar enough to a man.

As Pinto and Stein(2020) point out, the hard problem of consciousness, or the problem of explaining how (seemingly) immaterial experiences can arise from purely physical brain 
processes (Chalmers,1995), is the most important issue that any theory of consciousness should have a clear stance on (Pinto \& Stein, 2020). It has also been argued (Seth, 2016; Doerig et al., 2021) that it is reasonable to set the hard-problem aside and focus on conducting scientific experiments until we have a better understanding of consciousness. Considering how science works, if the game continues long enough then these two different strategies may potentially lead to the same outcome. However, I believe the former strategy (i.e. clarifying our position with regards to the hard-problem in particular and folk thinking in general) to be more efficient as there is no such thing as theory-laden observation. By the same token, the mere design of the experiments or the interpretation of the obtained data requires holding a plethora of assumptions over others. When those assumptions are not clear enough (i.e. we have not carefully considered why do we take them to be true) and in some cases even incompatible with one another, something that one researcher considers a confounding variable (e.g. reporting the conscious experience) might be perceived by others as essential (e.g. there is no conscious experience without conscious report). In some sense, avoiding the hard-problem and giving our tacit assumptions a free pass is what is already being done in the field, and when it comes to answering the most important questions (e.g. "if it is all physical, why does it feel different") we are almost back where we were decades ago. The job of consciousness studies is to actively tackle these problems, either by changing how we think about consciousness (and in case of failure) or by changing how we think about reality itself. Simply doing more of what we currently do does not seem to be getting the job done, beyond merely discovering facts about the brain. ${ }^{3}$

A good example of thinking differently about consciousness is Graziano's Attention-Schema theory of consciousness (Graziano 2016,2019,2020; Wilterson \& Graziano, 2021) which, while it borrows certain elements from GNWT, theory of mind and control theory, it does not make any unfalsifiable assumptions about the nature of consciousness, not does it rest upon folk notions such as a temporospatial onset. It is a theory about how various processes in the brain allow it to conclude it has consciousness and how this process can have evolutionary advantages. The theory also addresses why consciousness appears to have a non-physical quality to it from a subjective point of view. While the theory takes subjective perspectives on consciousness as data, it does not take them as assumptions. This is in sharp contrast with many other theories of consciousness that although eloquently describe processes in the brain, they still suffer from tacit folk assumptions about consciousness.

For instance, in GNWT, we are looking for that magical millisecond where we go from unconscious to conscious. This assumption is also further reflected in the concept of ignition, and the fact that a non-linear increase in the amplitude of certain Event-Related Potentials (ERP), that are stereotyped electrophysiological markers in response to certain stimuli, should be taken as neural correlates of consciousness (NCC). This is a classic case of mistaking the properties of the representee with the properties of the represented (Dennet, 1993). Indeed, there are studies (Windey \& Cleeremans, 2015) suggesting that

\footnotetext{
${ }^{3}$ This by no means is to diminish the value of discovering facts about the brain. Obviously, there will be no theory of consciousness without considering the facts about the brain. The point here is that having more empirical data does not equate to having better theories.
} 
consciousness may be binary with regard to some stimuli (i.e. high level stimuli), and continuous with regard to others (i.e. low level stimuli). However, the fact that consciousness sometimes "seems" ignited, does not necessitate a non-linear "jump" in the ERP signal simultaneously (Förster, Koivisto, \& Revonsuo, 2020).

It is precisely clinging on to such assumptions that slows down the course of our scientific progress by putting too much weight on never-ending "when/where" battles. A good example of this is the controversy around the "earliest onset of conscious experience", which is explained in great detail in Förster et al. (2020). As the name implies, the controversy is around finding out which ERP correlates better with the onset of conscious experience. There used to be three main camps on this issue:

1-The very early camp, identifying P1 component around $100 \mathrm{~ms}$ post-stimulus (Aru and Bachmann, 2009), following some studies that suggested a stronger P1 component in the aware conditions (Koivisto and Revonsuo, 2010).

2-The early camp, identifying visual awareness negativity (VAN) signal as the earliest onset of consciousness (Koivisto and Revonsuo,2003). VAN is calculated by subtracting the signal in the "aware" condition (i.e. when the participants report conscious awareness of the stimuli) from the "unaware" condition in any given task. The result is a small negative deflection in the signal at around $200 \mathrm{~ms}$ post stimulus, which is mostly visible in occipital areas and especially contralateral to the stimulus. (Jimenez et al., 2021)

3-The late camp, identifying P300 (more specifically, P3b) which occurs around 300 500 ms post-stimulus in parietal regions as the earliest correlate of consciousness.

Different theories of consciousness make different predictions in this regard. For instance, GNWT predicts a relatively "late" onset for consciousness, as it will take time for the information to reach the frontal regions and to become globally accessible to other parts of the brain. RPT, on the other hand, predicts a relatively "early" onset as in this framework, consciousness occurs as a consequence of a local occipito-temporal recurrent signal (Förster et al., 2020).

From a methodological perspective, it is fairly common for scientists to be critical of each other's theories. For instance, some studies suggest that P3b is confounded with the information being "task-relevant" or having to be "reported", thus it cannot reflect conscious activity per se (Förster et al., 2020; Ortego, Pitts, \& Cohen, 2020). On the other hand, some studies have failed to identify the VAN signal, while instead detecting the P3b (Salti et al. 2012; Lamy et al. 2008). Albeit, it has been argued that considering that VAN is a small deflection, it may require a study with high sensitivity for it to be detected (Koivisto and Grassini, 2016).

My issue with this debate however, is not a methodological one. Observing the evolution of this controversy, it seems that the "very early" position has been abandoned by its 
proponents, in light of new evidence (Förster et al., 2020). The issue with this debate is that what seems to be changing in this conversation is only the "magical millisecond" among the available options, after which the lights go on. This is merely a surface level change as this whole debate still assumes that there exists such a special state called consciousness, and that state has a beginning, and our job is to find that beginning.

Here one might object that even if a single onset for consciousness turns out to be an incorrect assumption, why would I claim that such research is misguided? After all, just like how we have managed to rule out the very early suggestion, we will ultimately rule out the other alternatives to get to the Truth, or that we will end up realizing that perhaps assuming a single onset for consciousness is incorrect. ${ }^{4}$

While this objection has its own merits, I provide two reasons as to why I am not fully convinced by it. First, there can be different ways to complete any given task but some ways are more efficient. For instance, using a brute-force strategy to sort a sufficiently large array of numbers should ultimately get the job done, but that does not mean one cannot accomplish the same functionality using faster and more efficient sorting algorithms. Similarly, we can spend decades of research and investigate all the candidate temporal and spatial onsets for "consciousness", or we can take all the current inconsistent empirical data to collectively suggest that we are asking the wrong kinds of questions. Furthermore, there are even empirical reasons to doubt a temporal onset for consciousness as this onset may be affected by expectation and contextual information (Melloni et al.,2011); however, most researchers have not included any experimental conditions to control for such confounds in their designs. This again shows how theories can shape empirical findings, and if future experiments confirm Melloni et al's findings, it can serve as a great example of how giving our assumptions a free-pass can slow down the course of actual progress.

Second, it seems that (in practice) no amount of scientific evidence in support or against any of these theories is ever enough to change the underlying assumptions nor to help us choose among different theories of consciousness. Using Kuhnian terminology, it is safe to say that current theories of consciousness are incommensurable; there is no straightforward way to rationally choose among them not only because there are valid empirical findings supporting them all, but also because different theories treat consciousness differently while referring to their explanandum as "consciousness". ${ }^{5}$ This is not an issue for most other fields of neuroscience as there are empirical and straightforward ways to choose among alternative theories.

\footnotetext{
${ }^{4}$ I thank an anonymous reviewer for pointing this out.

${ }^{5}$ My claim here is not that the scientific community cannot ever choose among different alternatives; rather, my claim is that we cannot do so "rationally". In principle, it should be possible to design experiments that favor one theory over the others. In practice, that experiment and the likes of it will be heavily criticized by the proponents of the opposing theory who will cite other empirically valid experiments supporting theirs. It is not immediately clear how empirical findings alone will necessarily resolve this controversy, without some form of collective value judgment among the experts. This is in contrast to the view that science is a measurable and linear progression towards the Objective Truth, independent of scientists themselves.
} 
The evolution of the majority of our models of consciousness seems to be partially driven by a collective cognitive dissonance; we have spent too much time building theories on top of our Cartesian and other folk assumptions that we find it hard to entertain the possibility that we may have been wrong all along. Instead, we procrastinate by picking another time window or another brain region to debate about, keeping our deepest assumptions away from scrutiny while at the same time, allowing ourselves to discover various facts about the brain and to create an illusion of progress.

Here, one might claim that all the new theories and constructs that we have so far amassed indicate actual empirical progress. However, having a growing list of constructs that refer to various neurophysiological processes does not necessarily mean that a scientific field is maturing similar to how knowing more words does not mean that one knows more concepts or can connect them efficiently to navigate one's environment.

To make matters worse, it seems that the proponents of different theories of consciousness, at least in some cases, may be ignoring the opposing theories, which is another unfortunate sign of incommensurability. As Del Pin et al (2021) point out, 93\% percent of published papers related to IIT or GNWT in the past decade, do not even mention the other theory. While all theories can be modified in light of new findings, for the field of consciousness studies to be truly able to address what it is expected to, we are in need of a Kuhnian paradigm shift ${ }^{6}$.

\section{Predictive Processes to the rescue?}

Recently, the conversations concerning the role of Prediction Error Minimization (PEM) frameworks have been gaining traction and it has been argued that theories of consciousness can benefit from incorporating PEM into their formulations (Hohwy \& Seth, 2020; Seth \& Hohwy, 2021; Walsh, McGovern, Clark, \& O'Connell, 2020). PEM paradigms treat the brain as a statistical inference device that consists of different levels in a hierarchical manner. Each level makes a prediction about the incoming bottom-up signal, and passes the difference (i.e. prediction error) to the level above with the aim of minimizing the prediction error both through cognition (e.g. selecting the top-down hypothesis that produces the least prediction error), and action (e.g. gazing towards a location where one expects the stimuli to appear). This is a vastly oversimplified account of the PEM paradigms and the topic has been explored thoroughly by Hohwy (2013), Clark (2015), Wiese and Metzinger (2017) among others.

It is not hard to appreciate the potential role a PEM framework can play in theories of consciousness considering the clear and robust accounts of various cognitive phenomena it brings to the table (Walsh et al., 2020). This is perhaps why Seth and Hohwy (2020) argue for incorporating PEM into existing theories of consciousness. However, the concept of NCC itself is also unclear, and can again be traced back to our metaphysical assumptions about

\footnotetext{
${ }^{6}$ For a recent review on Kuhn and paradigms, please see Anand, Larson, \& Mahoney (2020).
} 
consciousness. A systematic investigation of NCC only makes sense if one already assumes there exists a unique mental state, the consciousness, that if you exclude all the preconscious and post-perceptual activities in the brain as well as other possible confounds, then you are left with the minimum and pure neuronal regions and their dynamical interactions that explain consciousness (Koch, Massimini, Boly, \& Tononi, 2016). This seems to be another common misconception. As has been pointed out by Garfield (2016), you are never "just conscious", but that you are conscious of "something". In other words, there is no such thing as consciousness without content. ${ }^{7}$

Finally, Seth and Hohwy $(2020,2021)$ have gone to great lengths to explain how PEM is in fact compatible with some of our current theories of consciousness. The fact that PEM frameworks can link more local processes to more global ones in a hierarchical manner allows for other frameworks to adopt PEM regardless of whether they define consciousness locally or globally, at the bottom or at the top, early or late. While this highlights the importance of the PEM framework in a scientific account of consciousness and human cognition in general, the very fact that PEM can be incorporated in many other theories shows that it cannot be used to differentiate among them. All we can say is that given that the PEM framework offers a coherent account of brain function, it is therefore likely that a good theory of consciousness would include such a framework too. However, the ad-hoc inclusion of PEM does not seem to significantly improve the existing theories of consciousness.

\section{Can Illusionism give us the correct scientific assumptions?}

Illusionism is the idea that our subjective experience lacks phenomenal properties and thus, there is no explanatory gap ${ }^{8}$ once all the relevant brain processes are explained (Frankish, 2016). There are two important consequences that come with embedding theories of consciousness in Illusionist assumptions. First, is that given the Illusionist's rejection of the phenomenal aspect of subjective experience, an Illusionist theory does not have to make scientifically unfalsifiable assumptions (Dennett \& Cohen,2011). Second, and more importantly, Illusionism allows for the possibility to ask better questions, as it does not primarily emanate from folk notions about the mind $^{9}$. For instance, in an illusionist framework, the question of the earliest onset of consciousness would have no meaning whatsoever.

\footnotetext{
${ }^{7}$ There are indeed theories that treat consciousness separable from its contents, however, it is the general validity of those paradigms that is questioned here.

${ }^{8}$ The explanatory gap refers to the idea that no amount of knowledge about the physical world seems sufficient to account for why those processes are subjectively experienced in certain ways, or that they "feel like something" rather than nothing.

${ }^{9}$ It is logically possible to have an illusionist theory with incorrect assumptions, however, the essence of illusionist theories is more than simply denying phenomenal consciousness; it is doubting our subjective description of ourselves and the world including phenomenal consciousness. Therefore, an illusionist theory is less likely to make false assumptions based on folk concepts. This is not to dismiss all folk concepts as incorrect, but to accept them as initial inspiration for science and stories to be interpreted.
} 
This is not to imply that Illusionist assumptions about consciousness are therefore true; rather, it is a pragmatic solution for a scientific investigation of consciousness with clear metaphysical commitments. It may turn out that science can indeed never explain consciousness, or that we need to endorse new metaphysics such as panpsychism, but accepting this alternative before eliminating the possibility of Illusion is hasty and premature (Frankish, 2016).

Illusionism and its objections have been thoroughly addressed by Frankish and others (2016). Here, I will briefly respond to a recent objection raised by Brown (2021).

The author takes Descartes' second meditation to be the source of our "epistemic bedrock of certainty" and that if we somehow entertain the idea that our subjective experience is illusory, we will have no basis to rule out absolute nihilism:

If illusionism were true-or even possibly true - then we would lose our Cartesian bedrock of certainty in the existence of subjective experience. I believe that this would have an absurd consequence. Under the assumption that the only thing which we can be certain of is our current subjective experience, the even just-barely-possible truth of illusionism would entail that there is nothing we can be certain of. This means that it would be consistent with our epistemic situation that absolute nihilism - the view that nothing whatsoever exists (see Baldwin, 1996; Rodriguez-Pereyra,1997)-is possibly true. That is, Cartesian certainty in the existence of subjective experience forces at least one existential commitment on good epistemic agents: that there exists subjective experience. If the Cartesian view were false, it would be reasonable to entertain the possibility that absolute nihilism is true. Yet it is not reasonable to entertain the possibility that absolute nihilism is correct. So illusionism cannot possibly be true, since it entails an unacceptable epistemic conclusion.

--Brown (2021), p.2

While many thinkers may have strong feelings regarding Descartes, there are objections to this line of reasoning as well. Firstly, Descartes considers the possibility that even his mathematical knowledge could be somehow fed to him by an evil demon (Descartes, 1984/1641). But ultimately, in his quest to find what it is that he can be absolutely certain of, he uses a deductive argument. Where did that come from? It seems that one needs the assumption that "deduction must be true" to complete the meditation. How can " $2+2=4$ " be controlled by the evil demon, but not "If $A$ then $B$... A, therefore $B$ "? This is a contradiction, and it seems that Descartes' selection criteria for what to "doubt" are somewhat arbitrary. Secondly, and even granting the "deduction-smuggling" move, it seems that Descartes ended his line of questioning pre-maturely. One can conceive of a Modern Descartes $(M D)$, inspired by everything that has been discovered in the past four centuries, adding one final step to his meditation before concluding the famous cogito ergo sum. MD would think:

"... and finally, my subjective experience... Although it seems that my thoughts and my feelings contain a quality that I experience... A quality that cannot possibly be explained by any material science... this can be yet another trick by the evil demon... making me believe 
that I have experience... and yet even then, for the demon to deceive me into believing that there is a subjective feel to my experience... I must somehow exist to be tricked. "

MD's meditation contains everything in Descartes' meditation, it just does not take phenomenality as a metaphysical necessity. It is important to point out that this does not exclude the possibility that phenomenal properties can exist, it simply shows that what is central to Descartes' argument (even if Descartes himself thought otherwise) is not the existence of the phenomenal experience $X$ in a subject, but the existence of a subject capable of being deceived into believing anything, including the phenomenal experience of $X$. The argument works just as well with a zombie Descartes ${ }^{10}$.

\section{Final notes}

While there are numerous theories of consciousness, each praised for their unique handling of the empirical findings, the unfolding argument on one hand and tacit folk assumptions on the other, present serious challenges to their veracity. Although having unfalsifiable assumptions is a problem that some theories have to deal with, I believe this to be a symptom rather than the root of the problem. At the heart of the problem, is clinging on to a particular brand of assumptions and building theories around them, and a collective reluctance to update those core beliefs. This may perhaps be due to the fear that doing so will cause many of our current theories to fall apart. Yet, that is how revolutions in science occur and how science makes true progress. Given that all these theories explain various functions of the brain, it is unlikely that new and better models of consciousness would look entirely different from them. On the contrary, future theories will indeed inherit useful concepts from the past ones while leaving the incorrect aspects behind. AST inherits from the empirical findings and theoretical constructs from other theories, but treats them from a different theoretical perspective embedded in evolutionary biology. In addition to recent findings (Guterstam \& Graziano, 2020; Guterstam, Wilterson, Wachtell, \& Graziano, 2020; Wilterson et al., 2020) supporting this framework, there also has been an attempt to incorporate predictive processing in AST (Dołęga \& Dewhurst, 2019). These are all necessary characteristics that currently no other theory of consciousness possesses all at the same time, making AST a good candidate for an "obvious default theory of consciousness" (Dennett, 2016).

\section{Declaration of Competing Interest}

\footnotetext{
${ }^{10}$ A philosophical zombie is an imaginary being that is physically and behaviorally indistinguishable from a conscious person while it lacks phenomenal experience.
} 
The author declares that he has no known competing financial interests or personal relationships that could have appeared to influence the work reported in this paper.

\section{Acknowledgement}

I would like to thank Prof.Maria Falikman, Olesya Moiseenko, and the anonymous reviewers for valuable discussion and feedback.

\section{References}

Anand, G., Larson, E. C., \& Mahoney, J. T. (2020). Thomas Kuhn on Paradigms. Production and Operations Management, 29(7), 1650-1657.

Anderson, B. (2011). There is no such thing as attention. Frontiers in psychology, 2, 246.

Anscombe, G. E. M. (1959). An introduction to Wittgenstein's Tractatus.

Aru, J., \& Bachmann, T. (2009). Occipital EEG correlates of conscious awareness when subjective target shine-through and effective visual masking are compared: Bifocal early increase in gamma power and speed-up of P1. Brain research, 1271, 60-73.

Baldwin, T. (1996). There might be nothing. Analysis, 56(4), 231-238.

Block, N. (1995). On a confusion about a function of consciousness. Behavioral and brain sciences, 18(2), 227-247.

Brown, C. D. (2021). Why illusionism about consciousness is unbelievable. Ratio.

Chalmers, D. J. (1995). Facing up to the problem of consciousness. Journal of consciousness studies, 2(3), 200-219.

Clark, A. (2015). Surfing uncertainty: Prediction, action, and the embodied mind. Oxford University Press.

Cohen, M. A., \& Dennett, D. C. (2011). Consciousness cannot be separated from function. Trends in cognitive sciences, 15(8), 358-364.

Del Pin, S. H., Skóra, Z., Sandberg, K., Overgaard, M., \& Wierzchoń, M. (2021). Comparing theories of consciousness: why it matters and how to do it. Neuroscience of Consciousness, 2021(2), niab019.

Dennett, D. C. (1993). Consciousness explained. Penguin uk.

Dennett, D. C. (2016). Illusionism as the obvious default theory of consciousness. Journal of Consciousness Studies, 23(11-12), 65-72. 
Descartes, R. (1984/1641). Meditations on first philosophy. Caravan Books.

Dewhurst, J. (2021). Folk psychological and neurocognitive ontologies. In Neural Mechanisms (pp. 311-334). Springer, Cham.

Doerig, A., Schurger, A., Hess, K., \& Herzog, M. H. (2019). The unfolding argument: Why IIT and other causal structure theories cannot explain consciousness. Consciousness and cognition, 72, 49-59.

Doerig, A., Schurger, A., \& Herzog, M. H. (2021). Hard criteria for empirical theories of consciousness. Cognitive neuroscience, 12(2), 41-62.

Dolega, K., \& Dewhurst, J. (2019). Bayesian frugality and the representation of attention. Journal of Consciousness Studies, 26(3-4), 38-63.

Dołęga, K., \& Dewhurst, J. E. (2020). Fame in the predictive brain: a deflationary approach to explaining consciousness in the prediction error minimization framework. Synthese, 1-26.

Förster, J., Koivisto, M., \& Revonsuo, A. (2020). ERP and MEG correlates of visual consciousness: The second decade. Consciousness and Cognition, 80, 102917.

Frankish, K. (2012). Quining diet qualia. Consciousness and Cognition, 21(2), 667-676.

Frankish, K. (2017). Illusionism: as a Theory of Consciousness. Andrews UK Limited.

Garfield, J. L. (2016). Illusionism and givenness. Journal of Consciousness Studies, 23(11-12), 73-82.

Graziano, M. S. (2016). Consciousness engineered. Journal of Consciousness Studies, 23(1112), 98-115.

Graziano, M. S. (2019). Rethinking consciousness: a scientific theory of subjective experience. WW Norton \& Company.

Graziano, M. S. (2020). Consciousness and the attention schema: Why it has to be right. Cognitive Neuropsychology, 1-10.

Guterstam, A., \& Graziano, M. S. (2020). Visual motion assists in social cognition. Proceedings of the National Academy of Sciences, 117(50), 32165-32168.

Guterstam, A., Wilterson, A. I., Wachtell, D., \& Graziano, M. S. (2020). Other people's gaze encoded as implied motion in the human brain. Proceedings of the National Academy of Sciences, 117(23), 13162-13167.

Hohwy, J. (2013). The predictive mind. Oxford University Press.

Hohwy, J., \& Seth, A. (2020). Predictive processing as a systematic basis for identifying the neural correlates of consciousness. Philosophy and the Mind Sciences, 1(II).

Jimenez, M., Poch, C., Villalba-García, C., Sabater, L., Hinojosa, J. A., Montoro, P. R., \& Koivisto, M. (2021). The Level of Processing Modulates Visual Awareness: Evidence from 
Behavioral and Electrophysiological Measures. Journal of Cognitive Neuroscience, 33(7), 1295-1310.

Koch, C., Massimini, M., Boly, M., \& Tononi, G. (2016). Neural correlates of consciousness: progress and problems. Nature Reviews Neuroscience, 17(5), 307-321.

Koivisto, M., \& Grassini, S. (2016). Neural processing around 200 ms after stimulus-onset correlates with subjective visual awareness. Neuropsychologia, 84, 235-243.

Koivisto, M., \& Revonsuo, A. (2003). An ERP study of change detection, change blindness, and visual awareness. Psychophysiology, 40(3), 423-429.

Koivisto, M., \& Revonsuo, A. (2010). Event-related brain potential correlates of visual awareness. Neuroscience \& Biobehavioral Reviews, 34(6), 922-934.

Kukla, R. (2017). REALISM WITHOUT LITERALISM. The Philosophy of Daniel Dennett, 1.

Lamme, V. A. (2006). Towards a true neural stance on consciousness. Trends in cognitive sciences, 10(11), 494-501.

Lamy, D., Salti, M., \& Bar-Haim, Y. (2009). Neural correlates of subjective awareness and unconscious processing: an ERP study. Journal of cognitive neuroscience, 21(7), 1435-1446.

Mashour, G. A., Roelfsema, P., Changeux, J. P., \& Dehaene, S. (2020). Conscious processing and the global neuronal workspace hypothesis. Neuron, 105(5), 776-798.

Melloni, L., Schwiedrzik, C. M., Müller, N., Rodriguez, E., \& Singer, W. (2011). Expectations change the signatures and timing of electrophysiological correlates of perceptual awareness. Journal of Neuroscience, 31(4), 1386-1396.

Nagel, T. (1974). What is it like to be a bat. Readings in philosophy of psychology, 1, 159-168.

Oizumi, M., Albantakis, L., \& Tononi, G. (2014). From the phenomenology to the mechanisms of consciousness: integrated information theory 3.0. PLoS computational biology, 10(5), e1003588.

Ortego, K., Pitts, M., \& Cohen, M. (2020). Neural correlates of visual awareness and taskrelevance in a no-report masking paradigm. Journal of Vision, 20(11), 1600-1600.

Pinto, Y., \& Stein, T. (2021). The hard problem makes the easy problems hard-a reply to Doerig et al. Cognitive Neuroscience, 12(2), 97-98.

Rodriguez-Pereyra, G. (1997). There might be nothing: the subtraction argument improved. Analysis, 57(3), 159-166.

Salti, M., Bar-Haim, Y., \& Lamy, D. (2012). The P3 component of the ERP reflects conscious perception, not confidence. Consciousness and cognition, 21(2), 961-968.

Seth, A. K. (2016). The real problem. Aeon. Available online at: https://aeon. co/essays/thehard-problem-of-consciousness-is-a-distraction-from-the-realone (Accessed February 5, 2018). 
Seth, A. K., \& Hohwy, J. (2021). Predictive processing as an empirical theory for consciousness science. Cognitive Neuroscience, 12(2), 89-90.

Walsh, K. S., McGovern, D. P., Clark, A., \& O'Connell, R. G. (2020). Evaluating the neurophysiological evidence for predictive processing as a model of perception. Annals of the new York Academy of Sciences, 1464(1), 242.

Wiese, W., \& Metzinger, T. (2017). Vanilla PP for philosophers: A primer on predictive processing.

Wilterson, A. I., \& Graziano, M. S. (2021). The attention schema theory in a neural network agent: Controlling visuospatial attention using a descriptive model of attention. Proceedings of the National Academy of Sciences, 118(33).

Wilterson, A. I., Kemper, C. M., Kim, N., Webb, T. W., Reblando, A. M., \& Graziano, M. S. (2020). Attention control and the attention schema theory of consciousness. Progress in Neurobiology, 195, 101844.

Windey, B., \& Cleeremans, A. (2015). Consciousness as a graded and an all-or-none phenomenon: A conceptual analysis. Consciousness and cognition, 35, 185-191. 